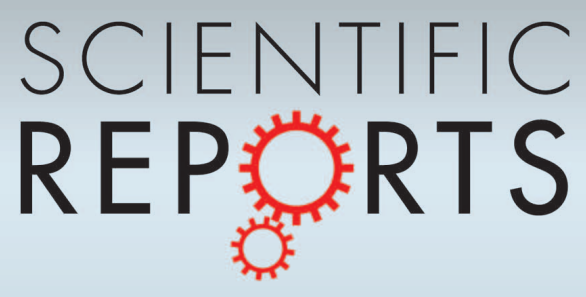

OPEN

SUBJECT AREAS:

ISCHAEMIA

HEART FAILURE

TRANSLATIONAL RESEARCH

DRUG DELIVERY

Received

5 June 2013

Accepted

13 February 2014

Published

7 March 2014

Correspondence and requests for materials should be addressed to

M.E.D. (michael. davis@bme.emory. edu)

\section{Targeting Extracellular DNA to Deliver IGF-1 to the Injured Heart}

\author{
Raffay S. Khan', Mario D. Martinez ${ }^{2}$, Jay C. Sy ${ }^{2}$, Karl D. Pendergrass', Pao-lin Che ${ }^{2}$, Milton E. Brown ${ }^{1,2}$, \\ E. Bernadette Cabigas ${ }^{2}$, Madhuri Dasari ${ }^{2}$, Niren Murthy ${ }^{2,3}$ \& Michael E. Davis ${ }^{1,2,3,4}$
}

\begin{abstract}
${ }^{1}$ Division of Cardiology, Emory University, Atlanta, Georgia 30322, USA, ${ }^{2}$ Wallace H. Coulter Department of Biomedical Engineering, Emory University and Georgia Institute of Technology, Atlanta, Georgia 30322, USA, ${ }^{3}$ Department of Bioengineering, University of California at Berkeley, Berkeley, CA, 94720, USA, 'Emory+Children's Center for Cardiovascular Biology, Children's Healthcare of Atlanta, Atlanta, GA 30332, USA.
\end{abstract}

There is a great need for the development of therapeutic strategies that can target biomolecules to damaged myocardium. Necrosis of myocardium during a myocardial infarction (MI) is characterized by extracellular release of DNA, which can serve as a potential target for ischemic tissue. Hoechst, a histological stain that binds to double-stranded DNA can be conjugated to a variety of molecules. Insulin-like growth factor-1 (IGF-1), a small protein/polypeptide with a short circulating-half life is cardioprotective following MI but its clinical use is limited by poor delivery, as intra-myocardial injections have poor retention and chronic systemic presence has adverse side effects. Here, we present a novel delivery vehicle for IGF-1, via its conjugation to Hoechst for targeting infarcted tissue. Using a mouse model of ischemia-reperfusion, we demonstrate that intravenous delivery of Hoechst-IGF-1 results in activation of Akt, a downstream target of IGF-1 and protects from cardiac fibrosis and dysfunction following MI.

D espite significant advances in therapy, a substantial proportion of patients treated for myocardial infarction (MI) develop congestive heart failure ${ }^{1}$. Recent breakthroughs in regenerative medicine with cell and growth factor based therapies show tremendous potential in reducing this risk. Current pharmacologic and device therapies however do not target damaged myocardium or recover functional myocardium, and rather serve to boost cardiac function and reduce inflammation. As damage from MI is mainly regional, localized therapy holds the most promise ${ }^{2}$.

Growth factor based therapeutics have shown great potential in regenerating the heart ${ }^{3}$. Insulin-like growth factor-1 (IGF-1) has demonstrated a cardio-protective and regenerative effect on the myocardium ${ }^{4}$. These benefits are primarily ascribed to the short-term actions of IGF-1 via activation of pro-survival pathways such as those mediated via Akt. In contrast, chronic exposure may be deleterious, associated with the development of pathologic cardiac hypertrophy and cancer ${ }^{5,6}$. Regulating the temporal effects of IGF-1 has been challenging, in part by its short circulating half-life, which has required prolonged infusions of growth hormone to raise IGF-1 levels, and its small size $(\sim 9 \mathrm{kDa})$, which results in poor tissue retention following direct injection ${ }^{7}$. For example, direct delivery of free IGF-1 to the infarcted heart does not allow for sufficient retention and does not lead to any improvement in cardiac function ${ }^{7}$. Thus, finding new ways to acutely localize IGF-1 delivery to the damaged myocardium could maximize its therapeutic benefits.

During an acute MI, excessive necrosis releases DNA in to the extracellular space. Given that nearly ten million cells show compromised cell membranes in rat models of $\mathrm{MI}^{8}$ this could mean on the order of picograms of DNA per cardiac cell. With estimates that scale rat to human studies demonstrating billions of myocytes potentially undergoing necrosis following acute MI, this represents a significant clinical target. In fact, high levels of circulating cell-free DNA have been found to correlate with myocardial necrosis in patients suffering from $\mathrm{MI}^{9}$ and other cardiovascular diseases such as peripheral artery disease, indicating other potential indications for using a DNA-targeting approach.

In this study, we demonstrate that IGF-1 conjugated to a DNA binding agent through streptavidin-biotin linkages can bind extracellular DNA and improve IGF-1 retention at sites of myocardial injury following MI. DNA binding dyes, such as Hoechst, have a high affinity for DNA $(\sim 60 \mathrm{nM})^{10}$ thus allowing them to localize to damaged myocardium by binding DNA released from dying cells. In addition, Hoechst has a history of being used safely in vivo as an angiogenic tracer and as a chemotherapeutic (topoisomerase inhibitor) in humans ${ }^{11,12}$. We 
A<smiles>[Y]C(C)(COC(=O)NC(C)(C)COc1ccc(-c2nc3cc(-c4nc5cc(N6CCN(C)CC6)ccc5[nH]4)ccc3[nH]2)cc1)NC(=O)CCCCC1SCC2NC(=O)NC21</smiles>

B

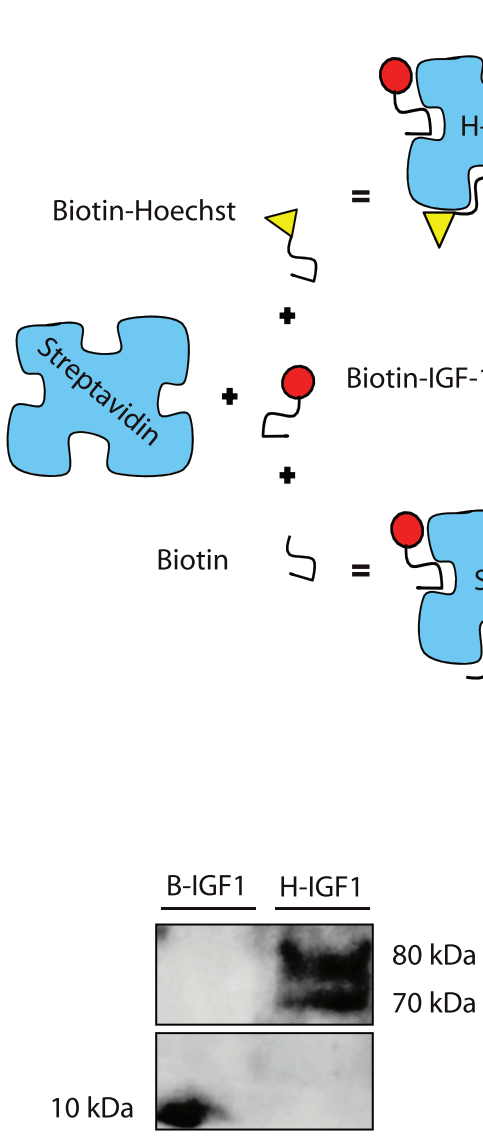

D

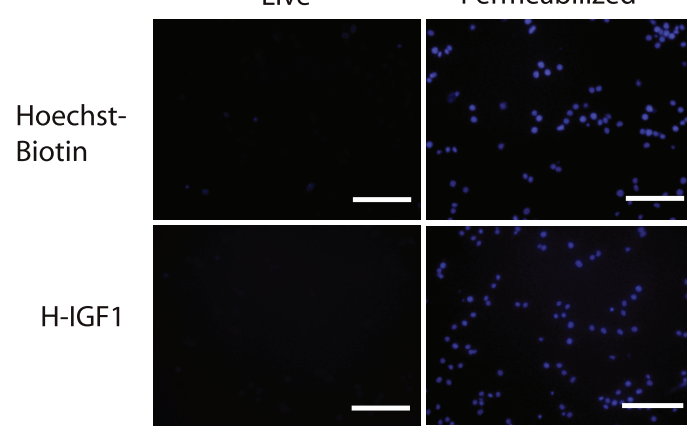

E

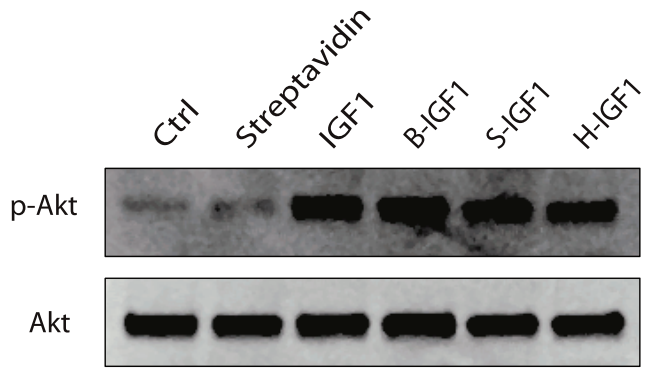

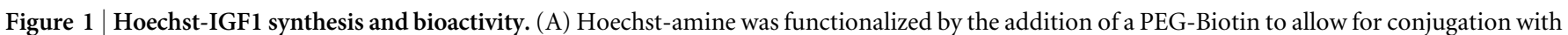

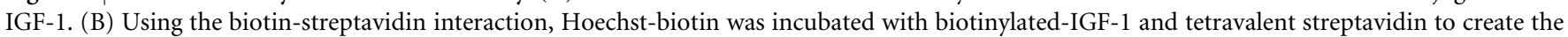
Hoechst-IGF1 (H-IGF1) complex. A control compound, streptavidin-IGF1 (S-IGF1) containing only streptavidin, biotin and biotinylated-IGF-1 was

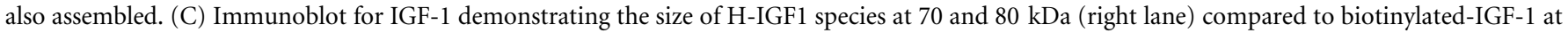
$10 \mathrm{kDa}$ (left lane). (D) Live and methanol fixed RAW 264.7 macrophages were treated with H-IGF1 or Hoechst-biotin for 15 minutes. Hoechst

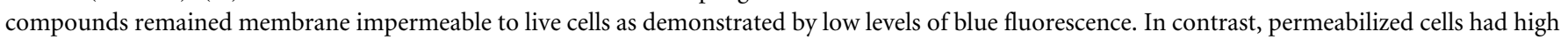
levels of blue, nuclear fluorescence demonstrating the DNA binding capability of Hoechst. White scale bar represents $100 \mu$ m. (E) Cardiomyoblasts (H9c2 cells) were treated with control media, streptavidin, IGF-1, biotinylated-IGF-1 or H-IGF1 for 15 minutes. Cells were homogenized and immunoblotted for Akt phosphorylation. H-IGF1 treatment activated Akt to a similar extent as IGF-1 and biotinylated-IGF-1.

therefore hypothesized that IGF-1 conjugation to Hoechst would allow IGF-1 to be delivered to areas of myocardial injury and attenuate MI-induced cardiac dysfunction and fibrosis.

\section{Results}

Creation and in-vitro validation of Hoechst-IGF-1. To conjugate IGF-1 to Hoechst, we used Hoechst previously modified by the addition of poly(ethylene glycol) (PEG) with a functional amine group $^{13}$ and further functionalized by the addition of a biotin moiety (Figure 1a). The structure of the final compound was verified through ${ }^{1} \mathrm{H}$-Nuclear magnetic resonance (Supplemental Fig. 1). Using biotin-streptavidin binding, biotinylated-human IGF-1 was then tethered to Hoechst-PEG-biotin to create HoechstIGF1 (H-IGF1), or attached to free biotin to make streptavidin-IGF1 (S-IGF1) as a non-targeted control (Figure 1b). Since each mole of streptavidin has 4 available binding sites for biotin, we expected Hoechst and IGF-1 to bind streptavidin in a $2: 2: 1$ molar ratio with the molecular weight of the final complex to be between 70 and $80 \mathrm{kDa}$. To confirm the size of the H-IGF1 complex and the presence of IGF-1, we immunoblotted against IGF-1. Two distinct 
bands for H-IGF1 were observed between 70 and $80 \mathrm{kDa}$, likely reflecting the different binding ratios of IGF-1 and Hoechst to streptavidin. As expected, biotin-IGF-1 was observed at $10 \mathrm{kDa}$ (Figure 1c).

An important consideration in localizing H-IGF1 to areas of myocardial damage is the selective targeting of DNA that is extracellular and ensuring that the H-IGF1 complex remains cell impermeable. We had previously shown that PEGylation and functionalization of the imaging agent, Hoechst-IR conferred membrane impermeability $^{13}$. To test if H-IGF1 behaved similarly, live and methanol fixed RAW 264.7 macrophages were incubated with H-IGF1 and imaged by fluorescence microscopy (Figure 1d). Treatment of live cells with either Hoechst-biotin or H-IGF1 demonstrated little to no nuclear fluorescence, indicating lack of cell permeability. In contrast, methanol-treated, permeabilized cells incubated with Hoechst-biotin or H-IGF1 generated high levels of intracellular fluorescence confirming that low intracellular fluorescence was due to the membrane impermeability of H-IGF1 and not due to a reduction in its DNA binding capability. Additionally, this also served to demonstrate that the functionalized Hoechst compounds could still bind DNA. Next, we confirmed the bioactivity of H-IGF1. Cardiomyoblasts (H9c2 cells) were quiesced in serum free media, washed and treated with control media, streptavidin or $50 \mathrm{ng} / \mathrm{ml}$ of IGF-1, biotinylated-IGF-1 or H-IGF1 for 15 minutes. As seen in the representative immunoblot, H-IGF1 phosphorylated Akt to a similar extent as both IGF-1 and biotinylated-IGF-1 (Figure 1e). These data demonstrate that both Hoechst and IGF-1 remain bioactive when conjugated to one another.

Intravenous administration of Hoechst-IGF-1 selectively binds exposed DNA and targets injured myocardium. To determine if Hoechst binds to DNA in areas of myocardial damage in vivo, we injected $500 \mu \mathrm{g}$ of Hoechst-biotin intravenously into rats subjected to ischemia-reperfusion (IR) or sham surgery. Confocal micrographs from hearts from sham animals demonstrated intact cell membranes in the left ventricle (LV) with minimal DNA binding, as evidenced by low levels of blue fluorescence. In contrast, IR hearts showed loss of membrane integrity with diffuse interstitial blue staining, indicating increased DNA binding (Figure 2a). Of note was the lack of any nuclear staining of Hoechst in sham or MI animals, indicating it did not enter intact, healthy cells. While rats were used to generate clear, morphologically distinguishable sections, all subsequent studies used mice.

DNA release from necrotic cells following MI typically occurs at 1-2 hours, peaks at $9-18$ hours and disappears by 72 to 96 hours $^{14}$. In order to ensure that an adequate amount of extracellular DNA was available to bind Hoechst, we subjected mice to 30 minutes of ischemia followed by reperfusion (IR) by ligating the left anterior descending artery and allowed 6 hours for sufficient necrosis to develop. Mice were then injected intravenously (tail vein) with either $110 \mu \mathrm{L}$ of PBS or equivolume amounts of S-IGF1 or H-IGF1 containing $200 \mathrm{ng}$ of IGF-1. At 2, 18 and 48 hours following injection, mice were sacrificed and organs harvested for imaging using a fluorescence imaging system (Figure 2b). The streptavidin linker used to create H-IGF1 and SIGF1 compounds contained a fluorophore, which was detected using a Cy5.5 filter. Fluorescence was then quantified to determine IGF-1 levels by plotting fluorescence intensity on standard curves generated by known amounts of IGF-1, as determined by ELISA (Supplemental Fig. 2). We specifically measured fluorescence in the LV, the major site of ischemic injury and DNA extrusion. Two hours following injection, there was an increase in fluorescence in the LV of both S-IGF1 and H-IGF1 treated animals, with no difference in IGF-1 levels between the two groups. This initial increase in IGF-1 levels likely resulted from passive accumulation of both H-IGF1 and SIGF1 due to enhanced permeability of the vasculature, a welldescribed phenomenon that typically occurs after ischemic injury ${ }^{15}$.
By 18 hours, LV IGF-1 levels from H-IGF1 treated mice increased by $27 \%$, accumulating $21.8 \%$ of injected dose per gram of tissue, second only to the liver. In contrast, LV IGF-1 levels from S-IGF1 treated mice decreased to $11.9 \% \mathrm{ID} / \mathrm{g}$ tissue, accumulating significantly less IGF-1 than the liver, spleen and lung (Figure 2c). IGF-1 levels in the liver, spleen, muscle and kidney were not statistically different between H-IGF1 and S-IGF1 treated mice. At 48 hours, IGF-1 levels in the LV from H-IGF1 treated mice began trending down but remained significantly greater than S-IGF1 treated mice. Additionally, no H-IGF1 was detected in blood samples at any of the 3 time points.

Many of the cardio-protective actions of IGF-1 are exerted via $\mathrm{Akt}^{7}$. We therefore measured the phosphorylation of Akt at the same time points used to determine IGF-1 targeting. In preliminary studies, we measured no significant changes over time in Akt phosphorylation in vehicle treated mice (Supplemental Figure 3). Similar to the temporal accumulation of IGF-1 in the LV, we found significantly greater phosphorylation of Akt in H-IGF1 mice over control but not S-IGF1 mice at 2 hours. However, by 18 hours, Akt activation peaked and was significantly greater in the LV of H-IGF1 treated mice than S-IGF1 mice. By 48 hours, Akt activation in H-IGF1 treated mice was reduced and was similar to levels seen in S-IGF1 treated mice (Figure 2d). Sham animals were used a control (dotted lines) to determine relative levels of increase. These data demonstrate that IGF-1 accumulation and activation of its downstream target occur in a time-dependent manner and parallel the accumulation of DNA into the extracellular space. To determine specificity for DNA, $\mathrm{H}-$ IGF-1 was preincubated with excess double-stranded DNA (1000×) prior to injection and imaging performed at 30 minutes. There was a significant decrease in localized H-IGF-1 that was preincubated with DNA as compared to H-IGF1 alone (Figure 2e).

Hoechst-IGF-1 administration in-vivo preserves cardiac function and prevents cardiac fibrosis following ischemia-reperfusion. In addition to determining IGF-1 targeting and retention in the LV, we also measured its functional impact. Cardiac function was assessed by echocardiography at 14 and 28 days following IR surgery and by invasive hemodynamics at 28 days. At both 14 (Supplemental Fig. 4) and 28 days, fractional shortening was greater in S-IGF1 and H-IGF1 treated mice compared to IR mice injected with vehicle. H-IGF1 treated mice however, demonstrated significantly greater cardiac contractility than S-IGF1 treated mice (Figure 3a). Moreover, HIGF1 treatment was associated with improved ejection fraction (Figure 3b), end-systolic (Figure 3c) and end-diastolic volumes (Figure 3d) as measured by invasive pressure-volume measurements. Given the improvement in cardiac remodeling with $\mathrm{H}$ IGF1 treatment, we hypothesized that increased IGF-1 delivery to the heart limited the development of cardiac fibrosis and reduced cardiac dysfunction. Histologic sections from 28 day-old mice were stained for collagen with Picrosirius red. Similar to the improvement in fractional shortening, both S-IGF1 and H-IGF1 treatments were associated with less cardiac fibrosis compared to control hearts, with H-IGF1 treated mice demonstrating markedly less cardiac fibrosis than S-IGF1 treated mice (Figure 3e).

\section{Discussion}

The ability to harness the promising potential of growth-factorbased therapy has been hampered by ineffective and potentially harmful delivery methods. In this report, we demonstrate for the first time that extracellular DNA released from necrotic cell death can be used as a target to deliver hormone therapy such as IGF-1. We show that a single intravenous injection of IGF-1 tethered to Hoechst results in increased IGF-1 localization to areas of cardiac injury with subsequent activation of Akt, through which many of its anti-apoptotic and cell proliferative effects occur. Whether this increase in IGF-1 accumulation results from greater Hoechst-mediated IGF-1 
A

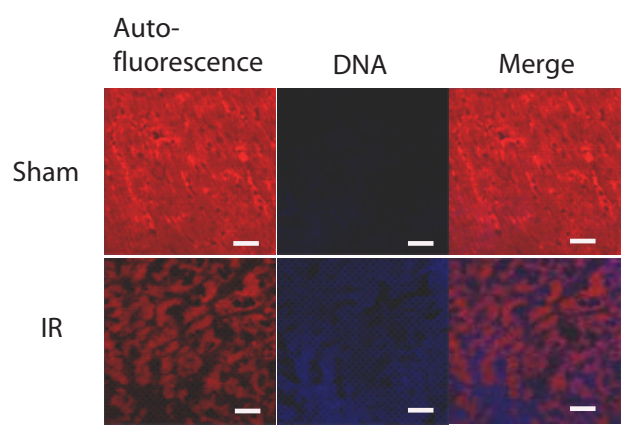

B
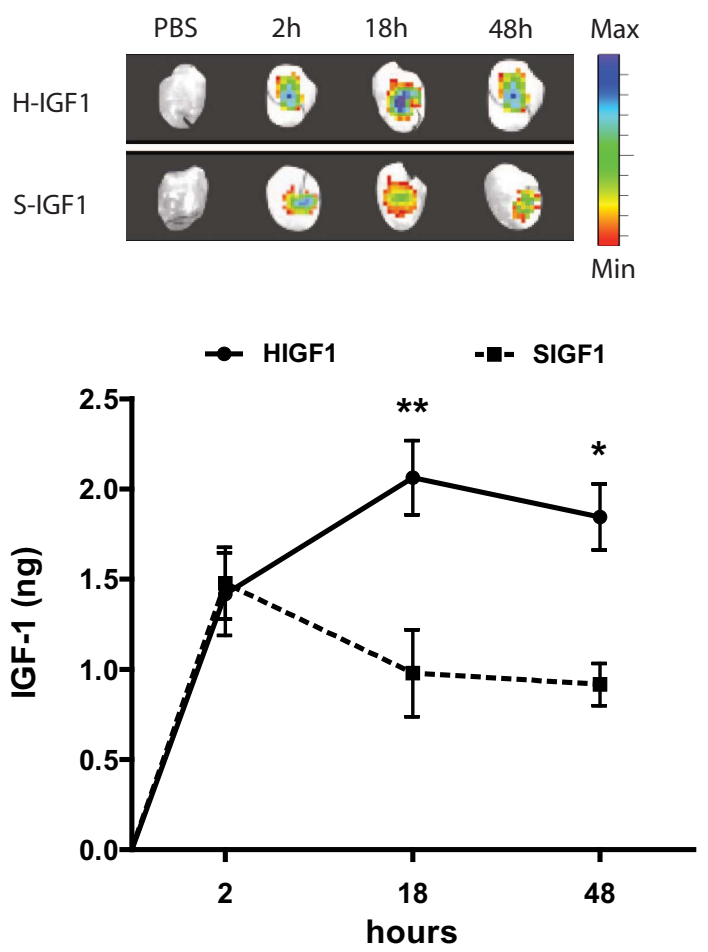

C
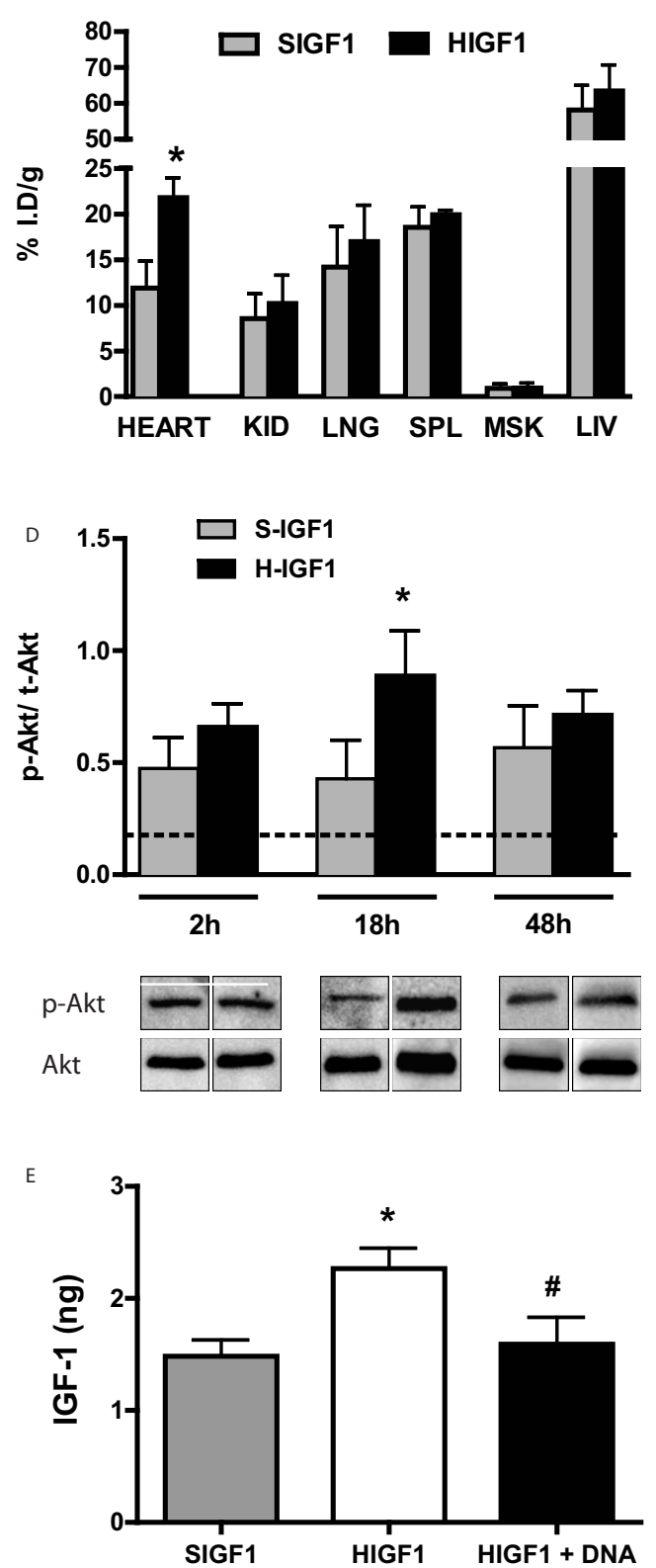

Figure $2 \mid$ Hoechst increases IGF-1 localization to infarcted myocardium. (A) Rats were subjected to ischemia-reperfusion (IR) surgery. One day later Hoechst-biotin was injected and allowed to circulate for $1 \mathrm{~h}$. Frozen sections were made and tissue was examined via red autofluorescence of the myocardium. Sham operated animals showed cell membrane integrity in the left ventricle (LV) and little blue fluorescence. IR-operated animals showed necrosis in the LV and diffuse blue staining suggesting release of DNA (Scale bar $=200 \mu \mathrm{m}$ ). (B) Imaging of hearts harvested from mice at the indicated time-point. Hoechst increased IGF-1 accumulation at 18 hours compared to S-IGF1 ( $\mathrm{n}=4-7$ per group; ${ }^{*} \mathrm{p}<0.05$ vs. S-IGF1, ** $\mathrm{p}<0.01$ vs. SIGF1, two-way ANOVA followed by Bonferroni post-test). (C) Imaging was used to quantify IGF-1 as \%ID per gram of tissue at $18 \mathrm{~h}$. While other organs were similar, there was a significant increase in the heart of H-IGF1 treated mice compared to S-IGF1 treatment ( $\mathrm{n} \geq 5$ per group, *p $<0.05$; t-test). (D) Western blots showing phosphorylation of Akt in the LV of mice treated with S-IGF1 or H-IGF1. By 18 hours, Akt phosphorylation in H-IGF1 treated mice was significantly greater than S-IGF1 treated mice. Line represents PBS treated IR mice. $\left({ }^{*} \mathrm{p}<0.05\right.$ vs. S-IGF1 at 18 hours, $\mathrm{n}=3-7$ per group, twoway ANOVA followed by Bonferroni post-test). (E) Mice were treated with S-IGF1, H-IGF1, or H-IGF1 pre-incubated with 1000× excess of doublestranded DNA. Pre-incubation with DNA significantly decreased IGF-1 levels. ( ${ }^{*} \mathrm{p}<0.05$ vs. S-IGF1, \# $\mathrm{p}<0.05$ vs. H-IGF1, $\mathrm{n}=5-7$ per group, one-way ANOVA followed by Bonferroni post-test).

delivery and subsequent DNA binding or is a consequence of reduced clearance of the Hoechst-DNA complex is unclear. Moreover, steric hindrance from the PEG-Hoechst chain could impede nucleases that normally degrade exposed DNA and contribute to increased retention of IGF-1 in the necrotic tissue. Finally, the addition of streptavidin (and PEGylated Hoechst) to IGF-1 may block the binding of IGF-1 binding proteins that are responsible for sequestering IGF-1 from the circulation. Despite these uncertainties, it is clear from the data that delivery of a growth factor with poor pharmacokinetics based on enhanced permeability is not effective.

We elected to use streptavidin-biotin chemistry to link Hoechst to IGF-1. This was based on our prior experience with this method to 
A

PBS

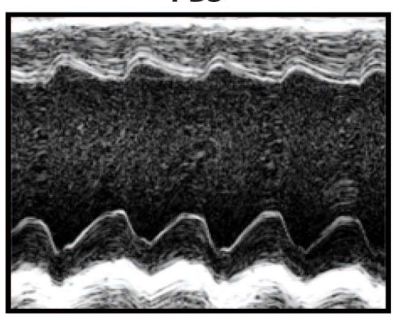

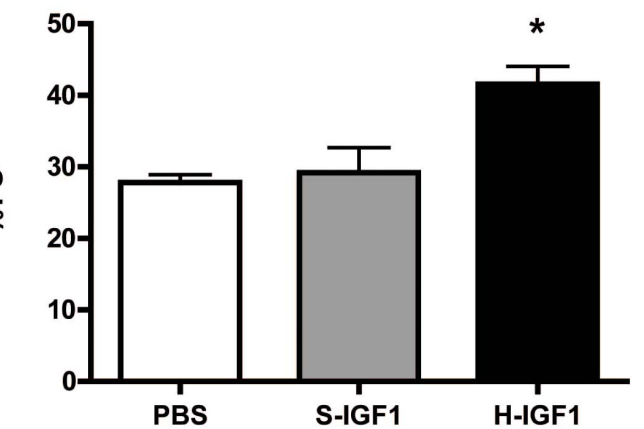

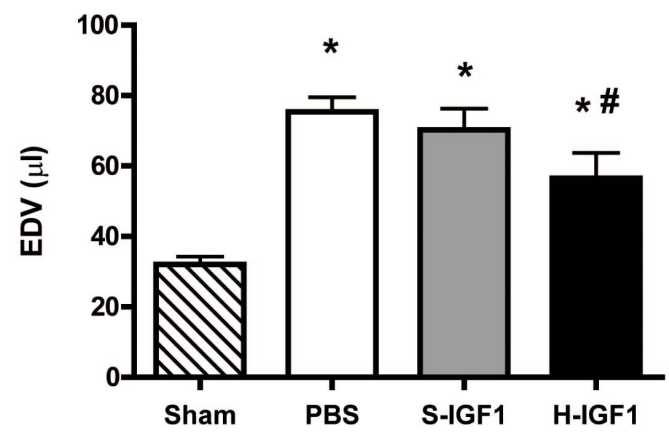

PBS

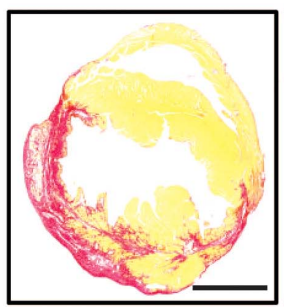

S-IGF1

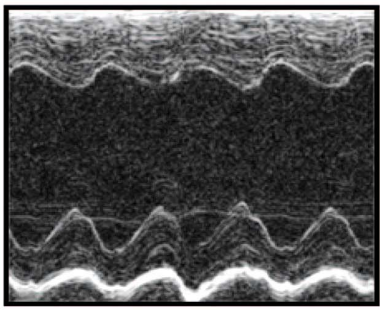

H-IGF1

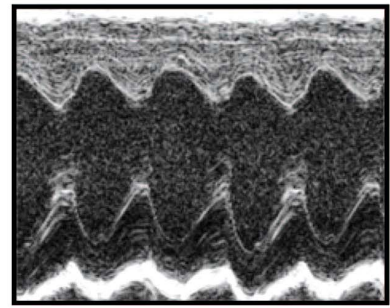

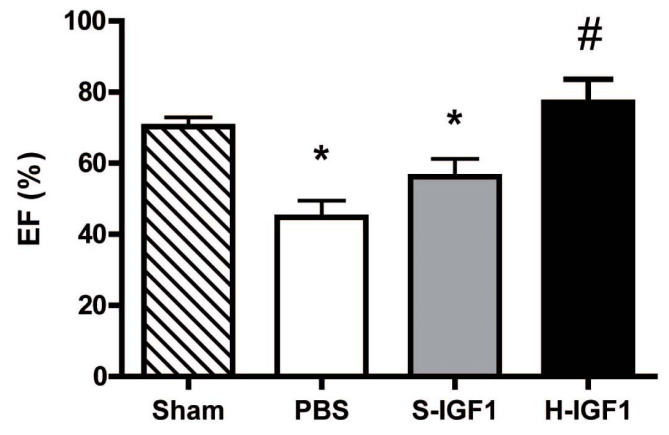
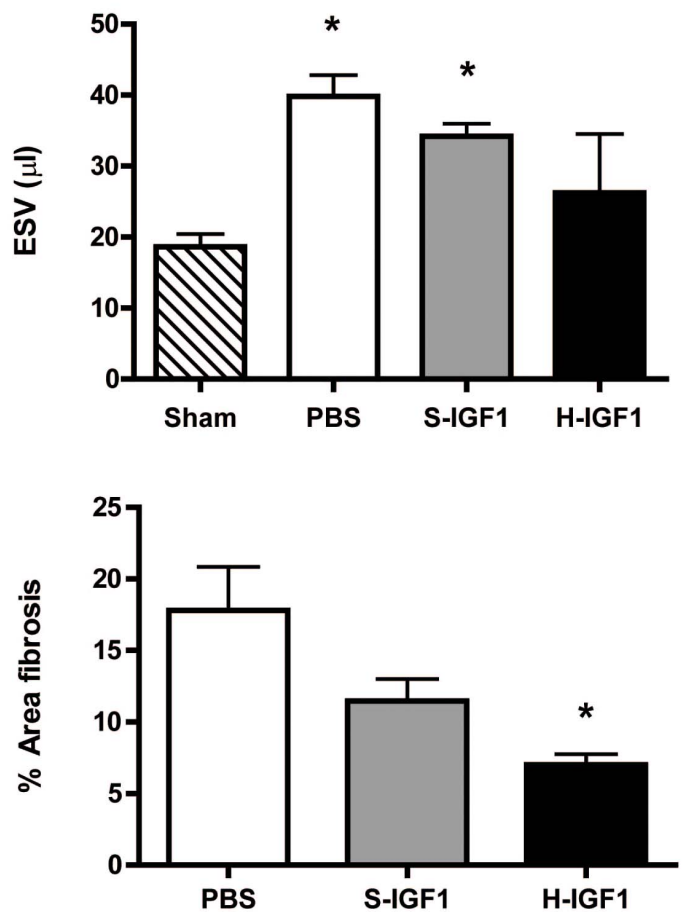

Figure 3 Hoechst-IGF1 preserves cardiac function and attenuates cardiac fibrosis following MI. (A) Representative echocardiographic M-mode images and bar graph depicting cardiac contractility at 28 days following IR. As shown, H-IGF1 treated mice demonstrated greater percent fractional shortening than PBS and S-IGF1 treated mice (\%FS, mean \pm SEM: $41.55 \pm 2.49$ vs. $29.18 \pm 3.51$ vs. $27.83 \pm 1.06) .{ }^{*} \mathrm{p}<0.01$ vs. S-IGF1, ** p $<0.001$ vs. PBS, $n=5-9$ per group, one-way ANOVA followed by Tukey's post-test. (B-D) Invasive cardiac hemodynamic measurements determined at 28 days following IR. H-IGF1 treatment led to significantly improved (B) Ejection fraction and (C) End-diastolic volumes compared to PBS and S-IGF1 treated mice. (D) End-systolic volumes were not different between sham and H-IGF1 treated mice ( $\mathrm{n}=5$ per group, ${ }^{*} \mathrm{p}<0.05 \mathrm{vs.} \mathrm{sham} \mathrm{mice,}{ }^{*} \mathrm{p}<0.05 \mathrm{vs}$. SIGF1 treated mice, one-way ANOVA followed by Tukey's post-test). (E) Representative Picrosirius red images from histologic sections of hearts from PBS, S-IGF1 and H-IGF1 treated mice. Black scale bar represents $1 \mathrm{~mm}$. H-IGF1 treatment reduced collagen content in the left ventricle compared to PBS treated mice ( $\mathrm{n}=5-6$ per group, ${ }^{*}, \mathrm{p}<0.05$ vs. PBS, one-way ANOVA followed by Tukey's post-test).

modify IGF-1, as well as the fact that our other Hoechst-reactive agents were primarily sulfhydryl-reactive ${ }^{7,13}$. IGF-1 has several cysteines that all are involved in complex disulfide bond formation which limits their use as reactive groups. Additionally, adding extra cysteines could interfere with protein folding and was not used. It is also likely that we had a heterogeneous mixture of species within our final formulation. While our Western blot data demonstrated a range of 1-2 IGF molecules per streptavidin, we were unable to determine how many Hoechst molecules there were per molecule of compound. Thus, due to incomplete binding or other reasons, there may have 
been some streptavidin molecules with 1 IGF molecule bound and a range of 1-3 Hoechst molecules (as streptavidin has 4 biotin binding sites). Additionally, while we dialyzed over several hours with large volumes of buffer, it is possible that not all unbound Hoechst-biotin was removed. While the affinity of biotin for streptavidin is high and we believe there were likely not many free Hoechst-biotin molecules, we cannot rule this out and it may compete with the H-IGF1 for DNA.

Our data is also consistent with previous reports of cardioprotection from IGF-1 therapy ${ }^{16,17}$. These beneficial effects have been attributed to Akt induced myocyte survival through an anti-apoptotic effect $^{18}$, angiogenesis ${ }^{19}$ and the protection of resident progenitor stem cells in the heart ${ }^{20}$. These data indicate that a single, systemic injection of H-IGF1 is able to preserve cardiac function and limit the development of cardiac fibrosis following MI. Moreover, we wish to point out that only $200 \mathrm{ng}$ of IGF-1 were injected, reducing the possibility for toxic effects seen at larger, prolonged doses ${ }^{5,6}$. With the broad chemistry surrounding functionalized PEG used to create Hoechst derivatives, it may be possible to target other therapies such as cells, small molecules, and even nanoparticles. We believe that the use of DNA binding agents such as Hoechst can be used to target exposed DNA in other diseases where necrotic cell death plays a critical role and could be used as a platform therapy.

\section{Methods}

Synthesis of hoechst compounds. Hoechst-amine was synthesized as previously described $^{13}$. Hoechst-biotin was synthesized from Hoechst-amine using NHS/EDCcoupling chemistry. EZ-link NHS-PEG12-Biotin was purchased from Thermo/ Pierce. Twenty-five milligrams of Hoechst-amine was dissolved in $5 \mathrm{ml}$ of anhydrous dimethyl formamide (DMF) with 20 equivalents of triethylamine under nitrogen). One equivalent of NHS-PEG compound was added to the reaction mixture and stirred overnight. Progress was monitored using TLC (MeOH:DCM). Upon completion of the reaction, DMF was removed under reduced pressure and the reaction mixture dissolved in $20 \%$ methanol (MeOH) in DCM with $0.5 \%$ triethylamine. This mixture was streaked on preparative TLC plates and developed using $10 \% \mathrm{MeOH}$ in DCM with $0.5 \%$ triethylamine. The product band was identified using a UV lamp and scraped off and MeOH:DCM used to extract the product.

$\mathrm{H}-\mathrm{IGF} 1$ was then generated using biotin-streptavidin chemistry. Since Streptavidin has 4 potential binding sites, Hoechst-biotin, human biotinylated-IGF-1 (immunological and biochemical test systems) and Streptavidin (labeled with a fluorescent tag, Thermo-Scientific Dylight 650) were combined in a 2:2:1 molar ratio for 3 hours at $37^{\circ} \mathrm{C}$ in PBS. The resulting mixture was dialyzed against PBS $(1000 \times)$ for 3 hours $(3$ changes, 1 hour each) to remove any unbound biotinylated-IGF-1 and Hoechstbiotin. The remaining sample was then extracted from the dialysis chamber and stored in $-20 \mathrm{C}$. The final concentration of IGF-1 of H-IGF1 and S-IGF1 conjugates was measured using a human-specific IGF-1 ELISA kit (VWR) as per manufacturers' instructions.

RAW 264.7 cells. RAW 264.7 macrophages were incubated with either HoechstBiotin or H-IGF1 $(20 \mu \mathrm{M})$ in DMEM containing $10 \%$ serum for 30 minutes at $37^{\circ} \mathrm{C}$. The cell media was then replaced by warm Krebs-Ringer buffer and the cellular fluorescence was recorded using a fluorescence microscope (Nikon E600, Nikon, Melville, NY), using an excitation wavelength of $340 \mathrm{~nm}$ and an emission filter between 400-500 nm. In case of fixed cells, RAW 264.7 macrophage cells were treated with pre-cooled methanol at $-20 \mathrm{C}$ for 10 minutes. The cells were washed with PBS followed by incubation with H-IGF1 for $30 \mathrm{~min}$ at $37 \mathrm{C}$. The cell media was then replaced by Krebs-Ringer buffer and the cellular fluorescence was recorded using a fluorescence microscope, with an excitation wavelength of $340 \mathrm{~nm}$ and an emission filter between $400-500 \mathrm{~nm}$.

Cardiomyoblast culture. H9c2 cells were purchased from ATCC and were plated at confluence 24 h before experiments in Dulbecco's Modified Eagle Medium containing l-glutamine, penicillin/streptomycin, and $2 \%$ fetal bovine serum. Media was then aspirated and replaced with treatment media (serum-free) containing either $50 \mathrm{ng} / \mathrm{mL}$ of IGF-1, B-IGF1, H-IGF1, Streptavidin or equal volume of PBS. After 15 minutes, media was aspirated and cells homogenized for protein extraction.

Myocardial infarction. Adult male mice 8 to 12 weeks old, fed an alfalfa-free diet were subjected to myocardial infarctions (MI) by ischemia-reperfusion (IR) surgery in a randomized and double-blind manner. Briefly, the animals were anaesthetized (1-3\% isoflurane, Webster Veterinary) and, following tracheal intubation, hearts were exposed by separation of the ribs. Myocardial infarction was induced by ligation of the left anterior descending coronary artery with a suture for a period of 30 minutes. The suture was then released and the heart reperfused. The chest was closed and the animals allowed to recover for $6 \mathrm{~h}$ to generate sufficient myocardial necrosis. After 6 hours, 110 ul of PBS, S-IGF1 or H-IGF1 were administered to mice via tail vein injection. Both S-IGF1 and H-IGF1 treatments were prepared in PBS and contained 200 ng of human-IGF-1 at 2, 18 and 48 hours, hearts were extracted, perfused with PBS and imaged directly under a fluorescence microscope (Xenogen IVIS 200 camera). All studies were performed in a randomized and blinded manner and were approved by the Emory University Institutional Animal Care and Use Committee.

Fluorescence imaging. In vivo fluorescence imaging was performed with an IVIS 200 small animal imaging system (Xenogen, Alameda, CA). A Cy5.5 filter set was used for acquiring $\mathrm{H}$-IGF1 and S-IGF1 fluorescence in vivo. Identical illumination settings (lamp voltage, filters, f/stop, field of views, binning) were used for acquiring all images, and fluorescence emission was normalized to total efficiency. Images were acquired and analyzed using Living Image 2.5 software (Xenogen, Alameda, CA).

Echocardiography. Two-dimensional echocardiography was performed using a high-resolution imaging system with a $30-\mathrm{MHz}$ imaging transducer (Vevo 770; VisualSonics) as described previously ${ }^{21}$ in anesthetized mice. Briefly the mice were anesthetized with $1.5-2 \%$ isoflurane and thereafter maintained on $1-1.5 \%$ isoflurane throughout the procedure. Two-dimensional echocardiographic images were obtained and recorded in a digital format. A researcher blinded to the murine treatment group analyzed images off-line. Left ventricular end-diastolic dimension (LVDd) and left ventricular end-systolic dimension (LVDs) were measured. Percent fractional shortening (\%FS), which quantifies contraction of the ventricular wall and is an indication of muscle function, was calculated as \% FS = ([LVDd - LVDs]/ LVDd) $\times 100$.

Invasive hemodynamic measurements. Briefly, cardiac function was first assessed under anesthesia by small animal echocardiography (Vevo770R, Visualsonics). Short axis values of left ventricular end systolic (ES) and end diastolic (ED) dimensions were obtained and used for pressure-volume calculations. For invasive ventricular function measurements, animals were anesthetized with isoflurane and placed on a heating pad. The chest was opened and a 1-French conductance catheter (Millar Instruments, MPVS Ultra and SPR-838) was introduced apically into the left ventricle. Pressurevolume loops were acquired with a PowerLab data acquisition system and analyzed using the LabChart software package (Millar). Catheter was calibrated according to manufacturer's instructions. At the completion of the experiment, animals were euthanized and hearts were excised for fixation and embedding.

Collagen staining. Collagen deposition was determined by Picrosirius red (Sigma) staining, as previously described ${ }^{22}$. Briefly, $5-\mu \mathrm{m}$ tissue sections were stained with Picrosirius red and imaged using a light microscope. A researcher blinded to treatment measured total collagen area (red staining) and normalized to total LV area in 3 separate sections per animal using ImageJ (NIH website).

Western analysis. Total protein was extracted from frozen hearts as described ${ }^{23}$. Proteins were resolved by SDS-PAGE and electro-transferred onto a PVDF membrane (Millipore Corp., Bedford, MA). The following antibodies were used: phospho-Akt (Ser473), (Cell Signaling Technology, Danvers, MA), Protein detection was carried out with the appropriate horseradish peroxidase-conjugated secondary antibody and ECL Plus detection systems (Amersham Biosciences, Piscataway, N.J.) Densitometry of bands was quantified using ImageJ (NIH Website).

1. Subcommittee, A. H. A. S. C. a. S. S. Heart Disease and Stroke Statistics-2012 Update. Circulation 125, e2-e220 (2011).

2. Anversa, P. Myocyte death in the pathological heart. Circ Res 86, 121-124.

3. Davis, M. E., Hsieh, P. C., Grodzinsky, A. J. \& Lee, R. T. Custom design of the cardiac microenvironment with biomaterials. Circ Res 97, 8-15 (2005).

4. Padin-Iruegas, M. E. et al. Cardiac progenitor cells and biotinylated insulin-like growth factor-1 nanofibers improve endogenous and exogenous myocardial regeneration after infarction. Circulation 120, 876-887 (2009).

5. Duerr, R. L. et al. Cardiovascular Effects of Insulin-Like Growth Factor-1 and Growth Hormone in Chronic Left Ventricular Failure in the Rat. Circulation 93, 2188-2196 (1996).

6. Wu, Y., Yakar, S., Zhao, L., Hennighausen, L. \& D, L. Circulating insulin-like growth factor-I levels regulate colon cancer growth and metastasis. Cancer Res $\mathbf{6 2}$, 1030-1035 (2002).

7. Davis, M. E. et al. Local myocardial insulin-like growth factor 1 (IGF-1) delivery with biotinylated peptide nanofibers improves cell therapy for myocardial infarction. Proc Natl Acad Sci U.S.A. 103, 8155-8160 (2006).

8. Kajstura, J. et al. Apoptotic and necrotic myocyte cell deaths are independent contributing variables of infarct size in rats. Lab Invest. 74 (1996).

9. Shimony, A. et al. Cell free DNA detected by a novel method in acute ST-elevation myocardial infarction patients. Acute Card Care. 12, 109-111 (2010).

10. Weisblum, B. \& Haenssler, E. Fluorometric properties of the bibenzimidazole derivative Hoechst 33258, a fluorescent probe specific for AT concentration in chromosomal DNA. Chromosoma 46, 255-260 (1974).

11. Baraldi, P. G. et al. DNA minor groove binders as potential antitumor and antimicrobial agents. Med Res Rev. 24, 475-528 (2004).

12. Paku, S. \& K, L. Morphological aspects of angiogenesis in experimental liver metastases. Am J Pathol. 143, 926-936 (1993). 
13. Dasari, M. et al. Hoechst-IR: an imaging agent that detects necrotic tissue in vivo by binding extracellular DNA. Org Lett. 12, 3300-3303 (2010).

14. Huang, S. et al. Molecular MRI of acute necrosis with a novel DNA-binding gadolinium chelate: kinetics of cell death and clearance in infarcted myocardium. Circ Cardiovasc Imaging. 4, 729-737 (2011).

15. Torchillin, V. Passive and active drug targeting: drug delivery to tumors as an example. Handb Exp Pharmacol., 3-53 (2010).

16. Li, Q. et al. Overexpression of insulin-like growth factor-1 in mice protects from myocyte death after infarction, attenuating ventricular dilation, wall stress, and cardiac hypertrophy. J Clin Invest. 100, 1991-1999 (1997).

17. O'Sullivan, J. F. et al. Potent long-term cardioprotective effects of single low-dose insulin-like growth factor-1 treatment postmyocardial infarction. Circ Cardiovasc Interv. 4, 327-335 (2011)

18. Sukhanov, S. et al. Differential requirement for nitric oxide in IGF-1-induced antiapoptotic, anti-oxidant and anti-atherosclerotic effects. FEBS Lett 585, 3065-3072 (2011).

19. Dobrucki, L. W. et al. Analysis of angiogenesis induced by local IGF-1 expression after myocardial infarction using microSPECT-CT imaging. J Mol Cell Cardiol. 48, 1071-1079 (2010).

20. Lu, G., Ashraf, M. \& K, H. H. Insulin-like growth factor-1 preconditioning accentuates intrinsic survival mechanism in stem cells to resist ischemic injury by orchestrating protein kinase $\mathrm{c} \alpha$-erk $1 / 2$ activation. Antioxid Redox Signal. 16, 217-227 (2012).

21. Drosatos, K. et al. Inhibition of c-Jun-N-terminal kinase increases cardiac peroxisome proliferator-activated receptor alpha expression and fatty acid oxidation and prevents lipopolysaccharide-induced heart dysfunction. J Biol Chem. 286, 36331-36339 (2011).

22. Sy, J. C. et al. Sustained release of a p38-inhibitor from non-inflammatory microspheres inhibits cardiac dysfunction. Nat Mater. 11, 863-868 (2008)

23. Khan, R. S. et al. Adipose tissue inflammation and adiponectin resistance in patients with advanced heart failure: correction after ventricular assist device implantation. Circ Heart Fail. 5, 340-348 (2012).

\section{Acknowledgments}

These publications have been funded in whole or in part with the Federal funds from the National Heart, Lung, and Blood Institute, National Institutes of Health, Department of Health and Human Services, under Contract No. HHSN268201000043C to MED and NM, as well as a predoctoral fellowship from the American Heart Association to MDM (13PRE16980068).

\section{Author contributions}

R.S.K. performed all delivery studies, imaging studies, and analyzed functional data. J.C.S. and M.D. synthesized Hoechst-conjugates and performed some delivery studies. MEB performed all animal surgeries, echocardiography, and invasive hemodynamics. M.D.M. and E.B.C. assisted in delivery and imaging studies. P.C. and K.D.P. helped with experimental design and in vitro characterization. R.S.K., M.E.D. and N.M. wrote the manuscript, while J.C.S., R.S.K., M.E.D. and N.M. edited the manuscript.

\section{Additional information}

Supplementary information accompanies this paper at http://www.nature.com/ scientificreports

Competing financial interests: The authors declare no competing financial interests.

How to cite this article: Khan, R.S. et al. Targeting Extracellular DNA to Deliver IGF-1 to the Injured Heart. Sci. Rep. 4, 4257; DOI:10.1038/srep04257 (2014).

cc)(1) $(-)$ This work is licensed under a Creative Commons AttributionNonCommercial-NoDerivs 3.0 Unported license. To view a copy of this license, visit http://creativecommons.org/licenses/by-nc-nd/3.0 